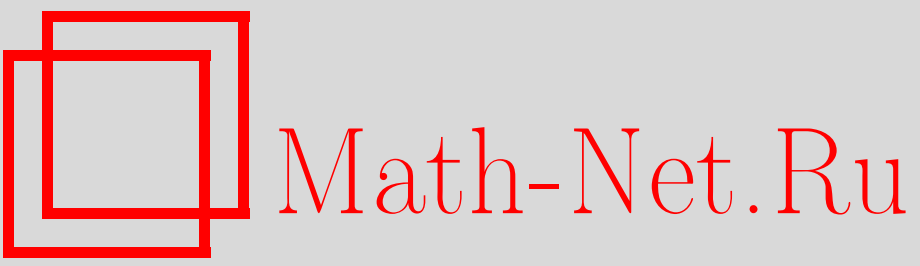

С. В. Людковский, Вложения неархимедовых банаховых многообразий в неархимедовы банаховы пространства, УМН, 1998, том 53, выпуск 5, 241-242

DOI: https://doi.org/10.4213/rm82

Использование Общероссийского математического портала Math-Net.Ru подразумевает, что вы прочитали и согласны с пользовательским соглашением

http://www.mathnet.ru/rus/agreement

Параметры загрузки:

IP: 54.198 .187 .58

26 апреля 2023 г., 16:41:00 


\title{
ВЛОЖЕНИЯ НЕАРХИМЕДОВЫХ БАНАХОВЫХ МНОГООБРАЗИЙ В НЕАРХИМЕДОВЫ БАНАХОВЫ ПРОСТРАНСТВА
}

\author{
С.В. Людковский
}

1. Определения. (а) Пусть $H$ - банахово пространство (БП) над нерхимедовым полем $\mathbf{K}$ [2], ультраметризуемое пространство $M$ назьвается многообразием смоделированным на $H$, если для $M$ выбран атлас $A t(M)=\left\{\left(U_{b}, f_{b}\right): b \in A\right\}$ с картами $\left(U_{b}, f_{b}\right)$ такими, что $f_{b}: U_{b} \rightarrow V_{b}$ - гомеоморфизмы, $U_{b}$ открыты в $M, V_{b}$ открыты в $H, \bigcup_{b} U_{b}=M ;$ (б) метрика $\rho$ на $M$ называется улштраметрикой, если $\rho(x, y) \leqslant \max (\rho(x, z) ; \rho(y, z))$ для любых $x, y, z \in M$; (в) микрорасслоением $X$ назьвается диаграмма $B \stackrel{i}{\rightarrow} E \stackrel{j}{\rightarrow} B$ состоящая из топологических пространств $B$ (базы) и $E$ (тотального пространства), а также непрерьвных отображений $i$ (инъекции) и $j$ (проекции) с $j \circ i=\mathrm{id}: B \rightarrow B$ - тождественньм отображением, причем выполнено условие тривиальности: $\forall z \in H \exists$ открытые $U \ni z$ и $V \ni i(z)$ с $i(U) \subset V, j(V) \subset U, h: V \rightarrow U \times H$ - гомеоморфизм, причем $j \circ i\left|U=p_{1} \circ x_{0}\right| U, h \circ i\left|U=x_{0}\right| U, p_{1} \circ h|V=j| V$, с инъекцией $x_{0}(u)=(u, 0)$, проекцией $p_{1}(u, x)=u($ см. [3] $)$. Далее $w(H)$ и $d(H)$ обозначают топологический вес и плотность соответственно для $H$.

2. Теорема. Если $M$ и $H$ из определений таковь, что $\operatorname{card}(A) \leqslant w(H), H$ бесконечномерно над $\mathbf{K}$ или $\mathbf{K}$ не локально компактно, то $M$ гомеоморфно открыто-замкнутому (ОЗ) подпространству в $H$.

ДокАЗАТЕльство. Пусть сначала $\mathbf{K}$ недискретно. Если $W^{*}=\left\{W_{q}: q \in J\right\}$ - открытое покрытие для $M$, то существует дизъюнктное покрытие $P^{*}$ ОЗ подмножествами $P_{s}$ вписанное в $W^{*}$ (см. 7.3 .15 в [4]). В самом деле, существует ультраметрика $\rho^{\prime}$ эквивалентная $\rho$ такая, что $\rho^{\prime}(x, y) / p \leqslant \rho(x, y) \leqslant p \rho^{\prime}(x, y)$ и $\rho^{\prime}(x, y) \in \Gamma(\mathbf{K}):=\left\{|x|_{\mathbf{K}}: x \in \mathbf{K}\right\}$ для любых $x, y \in M$. Шары $B_{\rho}(M, v, r):=\{y \in M: \rho(y, v) \leqslant r\}$ ОЗ в $M$ и любые два шара в $M$ либо не пересекаются, либо один из них содержится в другом в силу (1.б). Каждая характеристическая функция $h_{s}:=\chi_{P_{s}}: M \rightarrow \mathbf{K}$ непрерывна, следовательно, существует разбиение единицы $\left\{h_{s}: s\right\}$ подчиненное $W^{*}$. Тогда предложения 1,2 и 3 из [1] доказываются для неархимедовых $H$ (и микрорасслоений) вполне аналогично, причем они выполняются для ОЗ $U \supset i(B)($ в пр. 1) и $N \subset H$ (в пр. 2), где касательное расслоение $T M$ для $M$ класса гладкости $C^{t}$ с $t \geqslant 1$ определяется для недискретных полей $\mathbf{K}$ с помощью рассмотрения кривых $x: \mathbf{K} \rightarrow M$ и дифференцирований по параметрам, в частности, для $U_{b}$, а структурная группа является подгруппой в $G L(H)$ [5].

При доказательстве неархимедова варианта предложения $4[1]$ отрезок $I=[0,1] \subset \mathbb{R}$ можно заменить на компактньй единичный шар $B(L, 0,1)=: B^{\prime}$, где $L$ - локально компактное бесконечное подполе в $\mathbf{K}$, следовательно, $B^{\prime}$ имеет конечное подпокрытие $\left\{V_{y}: y\right\}$, возникающее из рассмотрения индуцированного микрорасслоения $b^{*}: H \times B^{\prime} \stackrel{\widetilde{i}}{\rightarrow} E^{*} \stackrel{\widetilde{j}}{\rightarrow} H \times B^{\prime}$. Тогда существует конечное семейство $t(0), \ldots, t(m) \in B^{\prime}, 1>r(k)>0$ такое, что $B^{\prime}=\bigcup_{k=0}^{m} B(L, t(k), r(k))$ - дизъюнктное объединение, а $b^{*} \mid U_{x} \times B(L, t(k), r(k))$ - тривиальны. Далее можно задать $R(0)=H \times\{0\}, R(q)=\left\{(x, t) \in U_{q} \times B^{\prime}:\left|\sum_{s<q} \lambda_{s}(x)\right|_{\mathbf{K}} \leqslant|t|_{\mathbf{K}} \leqslant\left|\sum_{s \leqslant q} \lambda_{s}(x)\right|_{\mathbf{K}}\right\}$, a потом рассуждать аналогично концу доказательства пр. 4 [1] трансфониинной индукцией по $q \in J$ вместо $i \in \mathbb{N}$ для семейства $\left\{Z_{q} \subset j^{-1}\left(\bigcup_{s \leqslant q} R(s)\right): q \in J\right\}$ упорядоченного по включению, а также варианта предложения 3 [1] для неархимедовых микрорасслоений, где $\left\{\lambda_{q}\right\}$ - разбиение 1 , соответствующее дизъюнктному ОЗ покрытию $\left\{U_{q}: q \in J\right\}, J$ - вполне упорядочено, 0 наименьший элемент в $J, \operatorname{card}(J)=w(H)$.

Вариант пр. 5 [1] для неархимедовых микрорасслоений выполняется также для ОЗ базы $N \subset$ $H$, a $W \supset i(N)$ может быть выбрана О3, так как при доказательстве достаточно рассмотреть ОЗ $W_{0} \subset \bigcup_{\alpha \in K^{0}} W_{\alpha}$. В самом деле, по теореме 3.8 [6] $H$ гомеоморфно $S^{\mathbb{N}}$, где $S$ - дискретное пространство мощности $\operatorname{card}(S)=w(H)$. Поскольку $w(B(H, v, r))=w(H) \geqslant \aleph_{0}$ для любых $v \in H$ и $r>0$, то из $d(\mathbf{K})=w(\mathbf{K}) \geqslant \aleph_{0}$ следует, что $d(H)=w(H)$ и существует разбиение пространств $X_{1}:=B(H, v, r), X_{2}:=B\left(H, v, r^{-}\right):=\{x \in H:\|x-v\|<r\}$ и $X_{3}:=H$ в дизъюнктные объединения ОЗ шаров, $X_{j}=\bigcup_{q \in L_{j}^{\prime}} B\left(H, v_{q}, r_{q}\right)$, мощности $\operatorname{card}\left(L_{j}^{\prime}\right)=w(H)$ 
для $j=1,2,3, r_{q}>0$ для любого $q$. Любые два ОЗ шара в $H$ гомеоморфны, следовательно, $X_{1} \cong X_{2} \cong X_{3}$ - гомеоморфны (для не сферически полного $\mathbf{K}$ шар $X_{2}$ открыт, но не замкнут в $H$ и в общем случае гомеоморфизмы $h_{j}: X_{2} \rightarrow X_{j}$ с $j=1$ или 3 не продолжаются на все $H$ ). Итак, для $N$ существует дискретное пространство $Y$ такое, что $N=Y \times H$, причем $Y$ можно рассматривать как объединение нульмерных одноточечных симплексов.

Далее рассмотрим изометричное вложение $(M, \rho)$ в свободное БП $B(M, \mathbf{K})$ (см. т. 5 в [7]). Тогда $d(B(M, \mathbf{K}))=w(B(M, \mathbf{K}))=d(M) d(\mathbf{K})=w(H)$, так как $d(M)=w(M)=$ $(\operatorname{card}(A)) d(H)$, следовательно, $B(M, \mathbf{K}) \cong S^{\mathbb{N}} \cong H$ - гомеоморфны и существует вложение $i: M \rightarrow H$. Пусть $M^{c}:=\left\{x \in H: \rho_{H}^{\prime}(x, M):=\inf _{y \in M}\|x-y\|_{H}^{\prime} \leqslant c\right\}$ обозначает $c$-раздутие $M \cong i(M)$ в $H$, где $c=c(n)=|\xi|_{\mathbf{K}}^{n}=p^{-n}, 1<p, \xi \in \mathbf{K}, n \in \mathbb{N},\|x\|_{H}^{\prime}:=$ $\inf _{b \geqslant\|x\|_{H}}\{b \in \Gamma(L)\}$ соответствует рассмотрению $H$ и $\mathbf{K}$ как БП над $L$, обозначаемых $H_{L}$ и $\mathbf{K}_{L}$ соответственно. Поле $L$ сферически полно, поэтому любое замкнутое $L$ - линейное подпространство $F$ в $H_{L}$ имеет ортогональное дополнение (в неархимедовом смысле) $H_{L} \ominus F$ (см. т. 5.13 и 5.16 [6]). Для каждого $M^{c}$ существует дизъюнктное ОЗ покрытие шарами в $H$. Если $v \in F \subset H_{L}$, то имеется ретракция $B(H, v, r)$ на $B(F, v, r)$ индуцированная проекцией $\pi: H_{L} \rightarrow F$. Поэтому, для любого $n \in \mathbb{N}$ существует ретракция $h_{n}: M^{c} \rightarrow M^{c / p}$ для $c=c(n)$, следовательно, $\left\{h_{n}: n\right\}$ дает ретракцию $r: M^{1} \rightarrow M$, так как $\bigcap_{n \in \mathbb{N}} M^{c(n)}=M$, где $r(x)=x$ для $x \in M, r(x)=h_{n}(x)$ для $x \in M^{c} \backslash M^{c / p}, M^{1}=: N$ ОЗ в $H$. Существует семейство подполей $L_{\beta}$ в $\mathbf{K}, \beta \in \Lambda$, такое, что $L_{\beta}$ - локально компактны, и минимальное поле порожденное $\bigcup_{\beta \in \Lambda} L_{\beta}$ плотно в $\mathbf{K}($ см. $\S \mathrm{X} .1[8])$.

В покрытие $\left\{U_{q}: q\right\}$ для $M$ можно вписать дизъюнктное ОЗ покрытие $W^{*}=\left\{W_{s}: s\right\}$ такое, что $W_{s}$ гомеоморфны ОЗ шарам в $H$. Тогда $W_{s} \times H \cong S^{\mathbb{N}} \cong H \times H \cong S^{\mathbb{N}} \cong W_{s}$ для любых $s$, следовательно, $M \times H \cong \bigcup_{s} W_{s} \cong M$. Рассматривая ОЗ $N$ и $W$ как и в доказательстве теоремы в [1] с использованием неархимедовых вариантов предложений 1-5 (см. выше), получим утверждение теоремы 2. В оставшемся случае дискретного $\mathbf{K}$ пространства $M$ и $H$ дискретны, так как $\{\rho(x, y): x, y \in M\} \subset \Gamma(\mathbf{K})$.

\section{СПИСОК ЛИТЕРАТУРЫ}

[1] Henderson D. W. // Topology. 1970. V. 9. P. 25-35. [2] Van Rooij A. C. M. Non-Archimedean functional analysis. New York: Marcel Dekker Inc., 1978. [3] Milnor J. // Topology. 1964-1965. V. 3. №1. Р. 53-80. [4] Энгелькинг Р. Общая топология. М.: Мир, 1986. [5] Бурбаки Н. Многообразия. М.: Мир, 1975. [6] Van Rooij A. C. M. // Report 7633. Math. Inst. Cathol. Univ., Nijmegen, The Netherlands, 1976. [7] Людковский С. В. // Фунд. и прикл. матем. 1995. Т. 1. С. 979-987. [8] Ленг С. Алгебра. М.: Мир, 1968. 